\title{
WHOLE BLOOD OR APHERESIS DONATIONS? A MULTI-OBJECTIVE STOCHASTIC OPTIMIZATION APPROACH
}

\begin{abstract}
In the blood supply chain, several alternative technologies are available for collection and processing. These technologies differ in cost and efficiency: for example, collection by apheresis requires very expensive machines but the yield of blood products is considerably greater than whole blood collection. Blood centre managers are faced with the difficult strategic problem of choosing the best combination of technologies, as well as the equally difficult operational problem of assigning donors to collection methods. These decisions are complex since so many factors have to be taken into account, including stochastic demand, blood group compatibilities, donor availability, the proportions of blood types in both donor and recipient populations, fixed and variable costs, and process efficiencies. The use of deterministic demand forecasts is rarely adequate and a robust decision must consider uncertainty and variability in demand as well as trade-offs between several potentially conflicting objectives. This paper presents a multi-objective stochastic integer linear programming model to support such decisions. The model treats demand as stochastic and seeks to optimize two objectives: the total cost and the number of donors required. To solve this problem, we apply a novel combination of Sample Average Approximation and the Augmented Epsilon-Constraint algorithm. This approach is illustrated using real data from Bogota, Colombia.
\end{abstract}

Keywords: OR in health services, Blood supply chain, Blood fractionation, Apheresis, Stochastic programming, Multiple objective programming

\section{Introduction}

The blood supply chain comprises the processes of collecting, testing, processing and distributing blood and blood products, from donor to recipient. Blood products are transfused to patients as part of routine medical treatments or surgical operations, and also in emergency situations. However, the increasing demand for blood products as well as the decreasing population of donors makes decision-making for the blood supply chain challenging (Seifried et al., 2011), and this is particularly the case in developing countries with limited resources. On the other hand, shelf-life constraints, multiple products, compatibilities and blood proportions make the problem complex, limiting the set of methodologies suitable.

Different configurations of the blood supply chain can be found in developed and developing countries. Developed countries tend to have centralized systems, while, in developing countries, the systems are 
often more decentralized. For example, in the UK there are five large production centres that supply blood for England and Wales (Woodget, 2014); in contrast, in Colombia there are 82 production centres of different sizes that provide blood products for the whole country. Another important difference between developed and developing countries is the availability of resources. According to the World Health Organization, the blood donation rate in high-income countries is 36.8 donors per 1000 population, while in middle-income and low-income countries it is 11.7 and 3.9 donations per 1000 population respectively (WHO, 2014). Hence, blood supply chain management is challenging in general; however, features such as economic resources, donor behavior and decentralization of the system have made these kinds of decisions even more challenging in developing countries.

A recent review (Osorio et al., 2015) of quantitative models in the blood supply chain identifies several gaps in the literature. One of the gaps identified is the necessity to study the different collection and production alternatives, given that blood products can be obtained in ways that differ in terms of cost and efficiency. Decisions about strategies to fulfil demand considering whole blood and apheresis donations have been rarely studied in general.

This paper contributes in two different ways. Firstly, the model proposed in this paper includes several characteristics that have not been taken into account in previous research in quantitative models for the blood supply chain, for example, the combination of uncertainty and multiple objectives simultaneously. Furthermore, the model includes other aspects that are rarely considered in blood supply chain literature such as multiple collection methods and multiple products simultaneously. Given a stochastic annual demand for blood products, the model supports strategic decisions such as technology selection and donor allocation and the use of substitute products in order to meet demand while minimizing both cost and the number of donors required. Secondly, in order to deal with uncertainty and multiple objectives, this work proposes a novel methodology that integrates two other approaches, namely Sample Average Approximation (SAA) and the Augmented Epsilon-Constraint algorithm. The model and the proposed methodology are evaluated using actual data in the public domain from Bogota, Colombia (INS, 2013).

This paper proceeds as follows: Section 2 presents the literature review of the main concepts used in this paper. Section 3 describes the problem and the type of decisions to be studied while Section 4 describes the data used. Section 5 introduces the mathematical formulation of the problem studied, with both a deterministic and a stochastic version of the model. Section 6 presents the integrated methodology proposed. Finally, in Section 7, the results of the application of the proposed model and methodology to a case study are presented, while Section 8 presents the main conclusions and extensions of this work. 


\section{Literature review}

\subsection{Quantitative techniques applied in the blood supply chain}

Research on the blood supply chain has been focused mainly on finding optimal inventory policies. Examples of this can be found in Pierskalla and Roach (1972), Cohen (1976), Nahmias and Pierskalla (1976), Chazan and Gal (1977) and Jagannathan and Sen (1991). An approach often used is simulation, which usually does not provide optimal solutions, but realistic policies and complex relationships can be studied using this technique. Examples of applications of simulation to the blood supply chain are provided by Rytilä and Spens (2006), Katsaliaki and Brailsford (2007), Baesler et al. (2011; 2013) and Simonetti et al. (2013).

One of the first papers on collection and production was presented by Cumming et al. (1976). In this work, forecasting models to improve collection goals and avoid over-collection were developed. On the other hand, survival analysis is used by Melnyk et al. (1995) to classify donors. Glynn et al. (2003) and Sonmezoglu et al. (2005) evaluate quality indicators for blood collected during disaster periods. Boppana and Chalasani (2007) develop Markov chains to define optimal collection policies during emergencies. Madden et al. (2007) compare efficiency indicators using both red blood cells obtained by fractionation and red blood cells obtained from the apheresis process. Lowalekar and Ravichandran (2010) develop a simulation model to evaluate several collection policies. In Arciniegas and Mosquera (2012) demand forecasts and a deterministic LP model are proposed to assign donors to collection processes over a period of 5 days. However, capacity and uncertainty are not considered. Alfonso et al. (2012; 2013) present two recent studies on this topic: capacity processing and staff required were estimated using Petri Nets and discrete event simulation. In Osorio et al. (2014) a deterministic model to define the collection strategy is proposed. The model presented in the current paper contains some similarities with the models presented in Osorio et al. (2014) and Arciniegas and Mosquera (2012). However, the main difference is that the model presented here considers uncertainty and stockouts, as well as the solution methodology developed. In general terms, unlike most of the literature described, this paper considers aspects of the blood supply chain such as uncertainty in demand, multiple collection methods, multiple products and multiple objectives.

\subsection{Multi-objective optimization: the augmented $\varepsilon$-constraint algorithm}

Several multi-objective optimization techniques have been proposed, such as weighted average methods, goal programming, $\varepsilon$-constraints and metaheuristics; a review of the main methods and applications can be found in Marler and Arora (2004) and Zhou et al. (2011). The best solution method for any particular problem depends on several factors including the complexity of the problem, the solution time and the required accuracy level of the solutions. However, in general terms, the aim of multi-objective optimization is to find a set of efficient solutions called the Pareto front. The Pareto 
front allows the decision-maker to have a wide overview of the behavior and trade-offs of the different objective functions.

One of the most frequently used methods for finding the Pareto front is the $\varepsilon$-constraint algorithm, first proposed by Haimes et al. (1971). This method converts multi-objective problems into single-objective problems by setting the objective functions as constraints bounded by epsilon parameters. The epsilon parameters are varied, thus obtaining the Pareto front. Examples of applications of this algorithm can be found in Bérubé et al. (2009) and Du et al. (2014). Improvements to the original method have been proposed in Ehrgott and Ruzika (2008) and Mavrotas (2009); the augmented $\varepsilon$-constraints algorithm due to Mavrotas is the version used in this paper. The application of the algorithm to the presented problem is explained in detail in Section 6.1.

\subsection{Sample Average Approximation}

SAA is one of the most widely used methods to deal with stochastic programming. In general, the expected value of the optimal solution for the stochastic problem is approximated using the average of samples obtained from probability distributions. According to Kleywegt et al. (2002), this method is designed for problems with three features. Firstly, the expected valued function cannot be easily calculated by analytical methods. Secondly, the value of the objective function can be easily calculated for an instance of the problem with stochastic elements. Finally, the set of feasible solutions is finite but considerably large, making enumeration approaches infeasible. The model presented in this article has these three features. Kleywegt et al. (2002) propose theoretical developments of the algorithm such as convergence rates, while applications can be found in Verweij et al. (2003) and $\mathrm{Li}$ (2014), including implementation aspects. In particular, applications in supply chains are described by Santoso et al. (2005), Schütz et al. (2009), Kiya and Davoudpour (2012), and Toro-Diaz and Osorio-Muriel (2012). Finally, applications in the blood supply chain are presented by Hemmelmayr et al. (2010) and Duan and Liao $(2013 ; 2014)$.

In the current paper the application of the SAA algorithm is combined with the multi-objective technique augmented $\varepsilon$-constraint algorithm in order to study the trade-off between multiple objectives in a stochastic optimization problem. A detailed explanation of the algorithm is presented in Section 6.2 .

\subsection{Combined multi-objective stochastic optimization}

There are many efficient methods to solve multi-objective problems and stochastic problems independently. However, according to Gutjahr and Pichler (2013), the combination of multi-objective stochastic problems has not been widely studied. Gutjahr and Pichler (2013) present a survey of the main types of problems, methods and applications. The literature regarding two-stage multi-objective stochastic optimization is sparse. In Fonseca et al. (2010) the objectives are combined in a single 
objective function and a set of scenarios is defined a priori. Cardona-Valdés et al. (2011) use the $\varepsilon$ constraint algorithm combined with the L-shaped method (also known as Benders' decomposition) to solve the problem using a set of scenarios also defined a priori. Finally, Tricoire et al. (2012) present a combination of the $\varepsilon$-constraint algorithm with a sampled-based method using a fixed random sample of the stochastic parameters; however, indicators of convergence are not evaluated. The methodology proposed in this paper differs from these papers in two main respects; firstly we use the augmented $\varepsilon$ constraint algorithm, which is an improved version. Secondly, we use the SAA algorithm to deal with the stochastic problem. This algorithm is sample-based and the solution is based not only on one sample of size $N$ but $M$ samples of size $N$. Although this algorithm is also sample-based, it is a more complex and rigorous approach than that used by Tricoire et al. (2012). This decreases the risk of omitting important scenarios.

\section{Problem description}

The most common blood collection method, called whole blood donation, consists of extracting approximately $450 \mathrm{~cm}^{3}$ of blood using a set of collection bags. The blood is centrifuged and, depending on the velocities and processing times, different components can be obtained using a process known as fractionation. The four main components derived from whole blood are red blood cells (RBCs), platelets, cryoprecipitate and plasma. On the other hand, apheresis processes, which directly withdraw a single blood component from a donor, are considerably more efficient than fractionation. For example, two units of red blood cells can be obtained from a donor using apheresis, while fractionation yields only one unit. However, apheresis also has some disadvantages. Firstly, it is considerably more expensive than fractionation and the donor time during this process is also longer. Secondly, donors must meet special conditions in terms of weight and hemoglobin levels in order to be able to donate RBCs by apheresis. Finally, only one product can be obtained from apheresis, which, depending on the nature of the demand, is not necessarily ideal.

Table 1 shows the five most common processes and the quantities of products obtained by assigning one donor to each process. The quantities are represented in standard transfusion units. One unit of whole blood collected in a triple bag yields one unit of RBCs and one unit of plasma. For quadruple bags, there are two fractionation alternatives: Alternative A generates one unit of RBCs, one unit of plasma and one unit of platelets, whereas Alternative B produces one unit of RBCs and one unit of cryoprecipitate. All three variants of whole blood collections can be processed on the same type of centrifuge machine, whereas the apheresis machines for collecting RBCs and platelets are different. 
Table 1. Quantity of blood products obtained by processes (standard units)

\begin{tabular}{|c|l|c|c|c|c|}
\hline No. & Process & RBCs & Plasma & Platelets & Cryoprecipitate \\
\hline 1 & Triplex bag & 1 & 1 & & \\
\hline 2 & Quadruple bag - Alternative A & 1 & 1 & 1 & \\
\hline 3 & Quadruple bag - Alternative B & 1 & & & 1 \\
\hline 4 & RBC by apheresis & 2 & & & \\
\hline 5 & Platelets by apheresis & & & 10 & \\
\hline
\end{tabular}

A further complicating feature of the blood supply chain is the possibility of using substitute products. Broadly speaking, blood types are defined in four groups (A, AB, B and $\mathrm{O}$ ), each of which is further subdivided by Rhesus factor (positive or negative). Compatibility between these eight blood types varies depending on the product to be transfused. Moreover, transfusion compatibilities can also vary according to factors such as physician preference, specific medical treatments and availability. The compatibility relationships used in this paper are presented in Appendix A.

The aim of the model proposed in this paper is to obtain a set of solutions that optimize both cost and the number of donors required, given uncertain demand. We illustrate this model by a case study that uses real data on donor supply and demand for blood products during one year in Bogota, Colombia. Cost information is based on the legal blood product list cost in Colombia, and commercial prices for the equipment.

\section{Case study background and data used}

The blood supply chain in Colombia consists of 82 blood banks and 414 transfusion services, which are distributed among 32 regions and the capital, Bogota. Bogota contains the largest number of blood centres in the same region, with 15 blood banks supplying blood products for 68 transfusion points; our case study is based on Bogota. In Colombia, the national blood bank network is controlled by the National Institute of Health, which defines legal aspects of the network, as well as the national blood policy. Nevertheless, in Colombia there is no common policy on collection processes, which means that that every region collects blood and blood products according to their own empirical decision rules. For example, in the Valle del Cauca region in 2012, 93\% of platelets were collected using apheresis processes while in the same period in the Antioquia region this percentage was just $42 \%$. However, most regions collect platelets from whole blood donations. In the case of RBCs, the greatest proportion of RBCs produced using apheresis processes is in the Tolima region with $8.24 \%$, followed by $6.26 \%$ from Bogota. Again, most regions obtain RBCs exclusively from whole blood donations.

The donation index (percentage of population donating) also varies throughout the country. While Bogota benefited from a donation index of $3.1 \%$ in 2012, the index for other Colombian regions was 
lower, for example, $2.5 \%$ and 2.2\% for Antioquia and Valle del Cauca respectively. More critically, the donation index was only $1.2 \%$ and $1.3 \%$ in the regions of Arauca and Nariño respectively, and these regions had to import blood products from elsewhere. Another performance indicator demonstrating problems in the supply chain is the amount received as a percentage of the amount ordered. In Bogota this rate was approximately $85 \%$ (probably caused by over-ordering in some periods), but in other regions, for example Norte de Santander, it was below $60 \%$. In general, this makes the donors a valuable and scarce resource in some regions. This situation is typical of low-income countries, where it is estimated that average blood donation rates are more than nine times lower than in high-income countries (WHO, 2016).

This case study utilizes real data concerning blood donor supply and transfusions performed in the city of Bogota in 2012. The main data source is published online by the Instituto Nacional de Salud (INS, 2013) and la Secretaria Distrital de Salud (SDS, 2013) and includes aggregated demand, donors and discard rates. The total demand in 2012 comprised 153,319 units of RBCs, 56,352 units of plasma, 91,815 units of platelets, and 14,511 units of cryoprecipitate. These figures are subdivided by blood groups and Rhesus factor according to historical rates. In 2012, a total of 222,954 RBCs were produced and 22,600 units were discarded for various reasons such as reactivity tests, expiration, and storage capacity.

The model requires data for all the different echelons of the supply chain. All blood collection centres routinely collect such data. At the collection stage, the model input data comprise historical numbers of donors, blood group rates, discard rates, cost of purchasing apheresis and fractionation equipment and the average collection time for each method. At the production stage, the model requires data on the efficiency of the production processes and the production costs. The model presented in this paper considers the 20 most common distinct products: 8 types of RBCs (by ABO group and Rh factor), 4 types of plasma and 4 types of cryoprecipitate (both by ABO group only), 2 types of fractionated platelets and 2 types of apheresis platelets (both by Rh factor only). At the inventory and distribution stages, it is necessary to know historical demand for each blood product by blood group. In our case study we used triangular distributions based on the Bogota demand data to take account of uncertainty, since sufficient data were not available to enable us to apply formal statistical procedures to fit probability distributions. The historical average demand for each blood product was used as the modal value, and the minimum and maximum values were simply $\pm 10 \%$ of the mode respectively. Integer values for demand were obtained by rounding up the sampled values from these triangular distributions.

\section{Mathematical model}

The integer linear programming model presented in this section optimizes two (competing) objective functions. On the one hand, total collection costs are minimized. On the other hand, the number of 
donors is also minimized. The decision variables are the numbers of machines to be purchased, the number of donors required, the collection/production strategy, and the policy for the use of substitute products. Other specific features of the blood supply chain, such as proportionalities of blood groups, multiple products, use of compatible products, and different collection methods, are either modelled as constraints or included in the definition of the decision variables.

\subsection{Problem formulated as a (deterministic) integer linear programming model}

We first present a deterministic version of the model. Although the formulation below is general, an explanation of the set indices and cardinalities for our particular case study example is provided to aid the reader's understanding.

Sets

$I=$ Blood types - indexed by $i$ and $a$. (ABO group and $\mathrm{Rh}+/-) .8$ in total.

$K \quad=$ Collection alternatives - indexed by $k$. (the rows in Table 1). 5 in total.

$J \quad=$ Products required - indexed by $j$ and $l .20$ in total.

$G \quad=$ Subset of K consisting only of alternative 4, "RBCs by apheresis" - indexed by $g .1$ in total.

$T \quad=$ Available technologies (machine types) - indexed by $t$. (Centrifuge for processing whole blood, and two different types of apheresis machine). 3 in total.

\section{Model Data}

$A_{i j k} \quad=$ Quantity of product type $j$ obtained by applying process $k$ to a unit of blood type $i, \quad i \in I$, $j \in J, k \in K$. [product units]

$P_{k} \quad=$ Production cost of processing one donation collected by alternative $k, k \in K .[\$]$

$F_{t} \quad=$ Fixed cost of purchasing one machine of type $t, t \in T$. [\$]

$C_{t}=$ Processing capacity (total time available) of one machine of type $t, t \in T$. [mins]

$R_{k t}=$ Time taken to process one donation collected by alternative $k$ using a machine of type $t$, $k \in K, t \in T$ [mins]

$D_{l} \quad=$ Demand for product $l, l \in J$. [product units]

$H_{l} \quad=$ Penalty cost for a stockout of product $l, l \in J .[\$]$

$O_{j l} \quad=1$ if product $j$ is substitutable with product $l, 0$ otherwise, $j, l \in J$.

$N_{i} \quad=$ Proportion of blood type $i$ in the population studied, $i \in I$.

$\alpha_{j} \quad=$ Historical discard rate of product $j, j \in J$. (explained in Section 4)

$B \quad=$ Proportion of population eligible for donation of RBCs by apheresis.

Decision variables

$x_{i k}=$ Target number of donors with blood type $i$ required for process $k, i \in I, k \in K$. 
$z_{j l} \quad=$ Quantity of product type $l$ that will be supplied by substitute product type $j, j, l \in J$.

$y_{t} \quad=$ Number of machines required of each technology type $t, t \in T$.

\section{Auxiliary variables}

$s_{l} \quad=$ Number of stockouts of product type $l, l \in J$.

\section{Model Formulation}

$$
\begin{aligned}
& \text { Minimise } R_{1}=\sum_{i \in I} \sum_{k \in K} P_{k} x_{i k}+\sum_{t \in T} y_{t} F_{t}+\sum_{l \in J} H_{l} s_{l}, \\
& \text { Minimise } R_{2}=\sum_{i \in I} \sum_{k \in K} x_{i k},
\end{aligned}
$$

subject to:

$$
\begin{aligned}
& \sum_{j \in J} z_{j l} O_{j l}+s_{l} \geq D_{l}\left(1+\alpha_{l}\right), \quad l \in J \\
& \sum_{l \in J} z_{j l} \leq \sum_{i \in I} \sum_{k \in K} x_{i k} A_{i j k}, \quad j \in J \\
& \sum_{k \in K} x_{i k} \leq N_{i} \sum_{a \in I} \sum_{k \in K} x_{a k}, \quad i \in I \\
& \sum_{i \in I} \sum_{k \in K} x_{i k} R_{k t} \leq C_{t} y_{t}, \quad t \in \mathrm{T} \\
& x_{i g} \leq \sum_{k \in K} x_{i k} B, \quad i \in I, g \in G \\
& s_{l}, x_{i k}, y_{t}, z_{j l}, \in \mathbb{Z}^{+} .
\end{aligned}
$$

The first objective function (1) represents the total annual cost $R_{1}$ of meeting the demand for blood products, including all variable and fixed costs. The second objective function (2) computes $R_{2}$, the total number of donors required to meet demand, considering all blood types and processes. Constraints (3) guarantee satisfaction of demand (increased by the discard rate) for each product, and constraints (4) define the quantity of product available as a function of the number of donors. Constraints (5) ensure that the proportionalities of each blood type in the population studied are conserved. The capacity constraints (6) define the availability of each resource according to the number of units of equipment for each technology. Finally, constraints (7) limit the number of donors that can be assigned to the process of producing RBCs by apheresis, since only a fraction of the population is eligible to donate by this method. 
Competition between objective functions (1) and (2) arises because there are many different ways to assign donors to collection method and these vary in terms of cost and the quantity of products obtained. The ranges for each objective function are given in Table 2, using values for three cases: the low, modal and high values of the triangular distributions used to model demand for blood products. For example, for one particular realization of demand (the modal value in Table 2), if we are interested only in minimizing costs and do not attempt to minimize donor numbers, then the minimum cost is $\$ 23.33 \mathrm{~m}$ and the required number of donors is 165,729 . On the other hand, for exactly the same demand realization, if we are not concerned about minimizing cost, the minimum number of donors required is only 137,352 but the costs increase to $\$ 30.36 \mathrm{~m}$.

Table 2. Extreme limits for each objective function.

\begin{tabular}{|l|l|c|c|}
\hline Scenario & Objective Function & Total cost & $\begin{array}{c}\text { Number of } \\
\text { donors }\end{array}$ \\
\hline \multirow{2}{*}{ Low } & Minimize cost $(\$ \mathrm{~m})$ & 22.18 & 157,466 \\
\cline { 2 - 4 } & Minimize number of donors & 28.88 & 130,483 \\
\hline \multirow{2}{*}{ Mode } & Minimize cost $(\$ \mathrm{~m})$ & 23.33 & 165,729 \\
\cline { 2 - 4 } & Minimize number of donors & 30.36 & 137,352 \\
\hline \multirow{2}{*}{ High } & Minimize cost $(\$ \mathrm{~m})$ & 26.84 & 190,600 \\
\cline { 2 - 4 } & Minimize number of donors & 34.85 & 157,951 \\
\hline
\end{tabular}

As shown in Table 2, the extreme lower and upper limits for the cost in the deterministic version of the problem are $\$ 22.18 \mathrm{~m}$ and $\$ 34.85 \mathrm{~m}$ respectively, while those for the donor objective function are 130,483 and 190,600. These values were calculated by solving the problem lexicographically, i.e. in order to calculate the maximum value for one objective we set the other objective as a constraint limited by its minimum value. However, general limits for the objective functions in the stochastic version of this multi-objective problem cannot be calculated directly (see further discussion in Section 6.3).

\subsection{Problem formulation as a two-stage stochastic optimization model}

In reality, demand is unknown, but decisions on the number of machines of each technology type to be purchased need to be made in advance, at the beginning of the planning horizon, before demand is realized. The challenge is to make good decisions at this stage that take into account the stochastic nature of the demand. In addition, a decision is made on the maximum number of donors to be recruited, so that later decisions do not require an unachievably large number of donors. The details on numbers of donors required by process and blood types are then determined after the uncertainty has been revealed. 
In order to take account of this uncertainty, the deterministic model in Section 5.1 can be restated as a two-stage stochastic linear programming model with recourse. The optimization then seeks optimal decisions for the first stage, based on the expected value of the second stage. The first stage of the problem is formulated as a multi-objective optimization problem in which we simultaneously minimise both the total expected costs and the maximum number of donors that will be allowed in the second stage. We add a new decision variable $q$ to represent this upper bound on the number of donors.

Model Formulation

$$
\begin{aligned}
& \min _{y, q} f(y, q)=\sum_{t \in T} y_{t} F_{t}+E[Q(y, q, \xi)] \\
& \min _{q} f(q)=q
\end{aligned}
$$

Subject to:

$$
y_{t}, q \in \mathbb{Z}^{+}
$$

The first objective function $f(y, q)$ represents the total expected cost. The decision variables are $q$ and the numbers $y_{t}$ of machines of each technology type $t$ to be purchased $(t \in \mathrm{T})$, where $F_{t}$ is the cost of one item of type $t$. The term $Q(y, q, \xi)$ denotes the optimal value of the second-stage stochastic singleobjective optimization problem, where $\xi$ is a random vector representing the stochasticity of demand. The second objective function, denoted by $f(q)$, is where we minimize $q$. The variable $q$ is fixed in the second stage problem as a upper limit on the number of donors required.

The term $Q(y, q, \xi)$ that is minimized in the second stage can be formulated as follows:

$$
Q(y, q, \xi)=\min \sum_{i \in I} \sum_{k \in K} P_{k} x_{i k}+\sum_{l \in J} H_{l} s_{l},
$$

subject to: (4) - (7), and a stochastic version of constraint (3):

$$
\sum_{j \in J} z_{j l} O_{j l}+s_{l} \geq D_{l}(\xi)\left(1+\alpha_{l}\right), \quad l \in J
$$

and

$$
\begin{aligned}
& \sum_{i \in I} \sum_{k \in K} x_{i k} \leq q, \\
& s_{l}, x_{i k}, z_{j l}, \in \mathbb{Z}^{+} .
\end{aligned}
$$


The objective function (12) calculates the variable cost of collection and the penalty for stockouts. The optimal set of solutions for the problem, $Q(y, q, \xi)$, is a function of the vector of first stage variables $y$ and $q$, and a particular realization of the random vector $\xi$. The second stage represents the decisions that can be modified according to demand realization, namely the number of donors required (by blood groups and collection methods), represented by the vector $x$, and the strategy for the use of substitute products, represented by the vector $z$. Constraint (13) limits the total number of donors, using the upper bound $q$, which was a decision variable in the first stage.

Uncertainty in the blood supply chain is mainly related to two sources, demand for products and donor arrivals. Demand for products depends on many factors such as surgeries, clinical treatments, emergencies and disasters. On the other hand, donation is a voluntary process that depends mainly on altruistic motivation. The uncertainty in donor arrivals is not modelled explicitly. However, we can confirm that the optimal target numbers of donors in the results are always lower than the actual numbers of donors observed in our data. It should also be noted that blood bank managers can use different strategies to increase blood collection when required. These strategies include targeted advertising to specific blood groups, special campaigns in popular places, and the use of frequent donor databases. In addition, in some cases blood bank managers ask relatives of patients to make donations in order to "replace" the blood used, although this is not desirable.

\section{Solution approach}

In order to solve the model presented in Section 5.2, we propose a novel approach which combines the SAA method and the augmented $\varepsilon$-constraint algorithm. The general idea is to solve one SAA problem for each epsilon value. The Pareto front thus generated is composed of the assigned value of one objective and the expected value of the other objective. The steps of this integrated approach are described below.

\subsection{Formulation of the augmented $\varepsilon$-constraint algorithm}

The $\varepsilon$-constraint technique proposed in Haimes et al. (1971) consists of converting a multi-objective problem into a single-objective problem. To do this, the decision-maker must pick one objective function to remain as the objective and set the others as constraints bounded by a set of parameters called epsilons. These parameters are defined individually for each objective converted into a constraint. In order to generate the Pareto front, the algorithm solves one single-objective optimization problem for each value of the $\varepsilon$ parameter. However, this form of the algorithm usually generates weak solutions, since the objective value could not be improved for some subsequent $\varepsilon$ values. In order to try to avoid this, and accelerate the whole process, Mavrotas (2009) proposes an improved version called the augmented $\varepsilon$-constraint algorithm (described in Appendix B). The formulation presented in Appendix 
B is general and considers $k$ objective functions; however, our model contains only two objective functions. Hence, normalization is not required. The number of models solved depends on how many epsilon values the decision-maker wants to analyse. As the increment of $\varepsilon$ becomes smaller, the algorithm will probably present a more detailed representation of the Pareto front but the computational cost will also be larger. Additional details of the algorithm can be found in Mavrotas (2009).

In order to apply the augmented $\varepsilon$-constraint algorithm to solve the problem presented in Section 5, different modifications are introduced in both the first and second stages. In the first stage, the second objective function $f(q)$ is converted into a constraint and is set equal to a parameter $\varepsilon$ as shown in Equation (15):

$$
q=\varepsilon
$$

By introducing these changes, the first-stage (multi-objective) problem can be reformulated as follows:

$$
\min _{y, q} f(y, q)=\sum_{t \in T} y_{t} F_{t}+E[Q(y, q, \xi)]
$$

subject to (11) and (15).

On the other hand, two main modifications are made to the second-stage model. Firstly, the objective function minimizing total costs is adapted by including a new surplus variable $w$ (described in Appendix B) as well as its coefficient $\beta$ :

$$
\text { Minimize }=\sum_{i \in I} \sum_{k \in K} P_{k} x_{i k}+\sum_{l \in J} H_{l} s_{l}-\beta w .
$$

Secondly, constraint (13) is modified as follows:

$$
\sum_{i \in I} \sum_{k \in K} x_{i k}+w=q
$$

By introducing these changes, the second-stage problem can be reformulated as follows:

$$
Q^{\prime}(y, q, \xi)=\min \sum_{i \in I} \sum_{k \in K} P_{k} x_{i k}+\sum_{l \in J} H_{l} s_{l}-\beta w
$$

subject to : (3'), (4) - (7), (14) and (17). 


\subsection{The Sample Average Approximation (SAA)}

The SAA method deals with uncertainty through the use of sampling. Hence the expected value $\mathrm{E}\left[\mathrm{Q}^{\prime}(\mathrm{y}, q, \xi)\right]$ of the second-stage objective function can be calculated by taking the average of the individual values of the samples of size $N$. In this case the resulting model is integer and linear. The whole problem can therefore be re-stated as follows:

$$
\min _{y \in Y}\left\{\hat{f}_{N}(y, q)=F y+\frac{1}{N} \sum_{n=1}^{N} Q^{\prime}\left(y, q, \xi^{n}\right)\right\}
$$

subject to $q=\varepsilon$, where $\varepsilon$ represents an upper bound on the number of donors.

The algorithm solves $M$ two-stage stochastic programming problems, each with $N$ scenarios (samples of the random vector $\xi$ ). Once a feasible first-stage solution $\{y, q\}$ has been found, an estimator for the true objective function value of the second-stage problem can be calculated, along with the optimality gap and its variance, using $N^{\prime}$ scenarios. This step involves solving $N^{\prime}$ deterministic second-stage problems. The complete SAA method (Santoso et al., 2005) is described as follows:

\section{$\underline{\text { Step 1: }}$}

Generate $M$ independent samples, each of size $N:\left(\xi_{j}^{1}, \ldots, \xi_{j}^{N}\right)$, for $j=1, \ldots, M$. For every sample solve the corresponding SAA (equation 19). Let $v_{N}^{j}$ and $\hat{y}_{N}^{j}$, for $j=1, \ldots, M$, represent the optimal value and optimal solution respectively.

$\underline{\text { Step 2: }}$

Calculate the following statistical indicators:

$$
\begin{aligned}
& \bar{v}_{N, M}=\frac{1}{M} \sum_{j=1}^{M} v_{N}^{j} \\
& \sigma_{\bar{v}_{N, M}^{2}}^{2}=\frac{1}{M(M-1)} \sum_{j=1}^{M}\left(v_{N}^{j}-\bar{v}_{N, M}\right)^{2}
\end{aligned}
$$

$\underline{\text { Step 3: }}$

Select a solution $\bar{y} \in Y$ of the original problem, using one of the $\hat{y}_{N}^{j}$ solutions already obtained. Estimate the true objective value $f(\bar{y}, q)$ by using the expression:

$$
\tilde{f}_{N^{\prime}}(\bar{y}, q)=F \bar{y}+\frac{1}{N^{\prime}} \sum_{n=1}^{N^{\prime}} Q^{\prime}\left(\bar{y}, q, \xi^{n}\right)
$$


where $\left(\xi^{1}, \ldots, \xi^{N^{\prime}}\right)$ is an independent sample of size $N^{\prime}$. It is expected that $N^{\prime}$ is considerably larger than the sample size $N$ used in step 1. Obtain the variance as follows:

$$
\sigma_{N^{\prime}}^{2}(\bar{y}, q)=\frac{1}{N^{\prime}\left(N^{\prime}-1\right)} \sum_{n=1}^{N^{\prime}}\left(F \bar{y}+Q^{\prime}\left(\bar{y}, q, \xi^{n}\right)-\tilde{f}_{N^{\prime}}(\bar{y}, q)\right)^{2}
$$

Step 4:

Calculate the optimality estimator based on the results from steps 2 and 3.

$$
\operatorname{gap}_{N, M, N^{\prime}}=\tilde{f}_{N^{\prime}}(\bar{y}, q)-\bar{v}_{N, M}
$$

Estimate the variance of the optimality gap using the expression:

$$
\sigma_{g a p}^{2}=\sigma_{N^{\prime}}^{2}(\bar{y}, q)+\sigma_{\bar{v}_{N, M}}^{2}
$$

The solution of the SAA problem presented in equation (19) can be challenging, depending on the nature of the variables and the size of the problem. Since using this approach for a multi-objective problem implies solving multiple SAA problems for different values of $\varepsilon$, the solution time for each individual SAA problem is critical. Therefore, an efficient solution approach for the SAA problem must be chosen carefully. In order to find a suitable method to solve the SAA problem rapidly, we investigated three different approaches. Firstly, the problem is solved considering $N$ scenarios simultaneously, keeping the integrality condition over all the decision variables. This approach corresponds to configurations 1-3 in Table 3. The second approach also considers $N$ scenarios simultaneously; however, in this case the integrality constraints for some of the decision variables are relaxed. In configurations 4-6 only the variables $w$ and $s$ are allowed to be continuous, whereas in configurations 7-9 all the integer variables are allowed to be continuous apart from the first-stage variables $y$. Integrality is restored in both cases by rounding up the fractional values. The third approach is different: unlike the first two approaches, we solve the SAA problem using decomposition rather than simultaneity. We apply Benders' decomposition algorithm (also known as the L-shaped method) to solve the SAA problem (see Appendix C). The results are presented in configurations 10-12.

In order to evaluate these solution strategies, we used the modal value of the triangular probability distribution of demand, and an arbitrary (but realistic) $\varepsilon$ value of 150,000 for the maximum number of donors. Table 3 presents the results for all 12 configurations in terms of convergence, solution time and sample size. The values obtained for the objective function and the convergence indicators for each solution method and set of configuration parameters are presented in Table 4. The size of the samples and the number of replications for the SAA were selected based on the literature (Verweij et al. (2003) and Santoso et al. (2005)). For our specific instance, the gap estimator for all cases is lower than $1 \%$, which is a good convergence indicator of the algorithm, and suggests that the chosen parameters are 
valid. Based on the solution time, the optimality gap, the size of the samples and the number of replications, we chose configuration number 9 as an initial configuration for all the $\varepsilon$ values; however, in general this choice could be modified if the optimality gap indicator were higher than some threshold value.

This configuration performed well for this specific problem; however, other alternatives could be used in the solution of the SAA problem such as acceleration techniques for Bender's decomposition, branch and price, and branch and cut as well as other solution approaches found in the literature such as Schütz et al. (2009) and Ogbe and Li (2017). 
Table 3. Summary of model features for different configurations of the SAA problem (see section 6.2 for the explanation of N, M and N')

\begin{tabular}{|c|c|c|c|c|c|c|c|c|c|c|c|}
\hline Configuration & $\mathrm{N}$ & M & $\mathrm{N}^{\prime}$ & $\begin{array}{l}\text { Solution } \\
\text { approach }\end{array}$ & $\begin{array}{c}\text { Variables with } \\
\text { integrality constraints } \\
\text { relaxed }\end{array}$ & $\begin{array}{l}\text { Variables with } \\
\text { integrality } \\
\text { constraints } \\
\end{array}$ & $\begin{array}{l}\text { Continuous } \\
\text { variables }\end{array}$ & $\begin{array}{c}\text { Integer } \\
\text { variables in } \\
\text { SAA problem }\end{array}$ & $\begin{array}{c}\text { Number of } \\
\text { constraints in } \\
\text { SAA problem }\end{array}$ & $\begin{array}{c}\text { Total number } \\
\text { of models } \\
\text { solved } \\
\end{array}$ & $\begin{array}{l}\text { Time } \\
\text { (sec.) }\end{array}$ \\
\hline 1 & 20 & 10 & 500 & Simultaneous & - & $\mathrm{y}, \mathrm{x}, \mathrm{z}, \mathrm{w}$ and $\mathrm{s}$ & 0 & 9223 & 1200 & 510 & 118 \\
\hline 2 & 40 & 10 & 500 & Simultaneous & - & $\mathrm{y}, \mathrm{x}, \mathrm{z}, \mathrm{w}$ and $\mathrm{s}$ & 0 & 18443 & 2400 & 510 & 340 \\
\hline 3 & 30 & 20 & 1000 & Simultaneous & - & $\mathrm{y}, \mathrm{x}, \mathrm{z}, \mathrm{w}$ and $\mathrm{s}$ & 0 & 13833 & 1800 & 1020 & 343 \\
\hline 4 & 20 & 10 & 500 & Simultaneous & $\mathrm{W}, \mathrm{S}$ & $\mathrm{y}, \mathrm{x}$ and $\mathrm{z}$ & 420 & 8803 & 1200 & 510 & 112 \\
\hline 5 & 40 & 10 & 500 & Simultaneous & $\mathrm{w}, \mathrm{s}$ & $\mathrm{y}, \mathrm{x}$ and $\mathrm{z}$ & 840 & 17603 & 2400 & 510 & 156 \\
\hline 6 & 30 & 20 & 1000 & Simultaneous & $\mathrm{w}, \mathrm{s}$ & $\mathrm{y}, \mathrm{x}$ and $\mathrm{z}$ & 630 & 13203 & 1800 & 1020 & 239 \\
\hline 7 & 20 & 10 & 500 & Simultaneous & $\mathrm{W}, \mathrm{s}, \mathrm{X}, \mathrm{Z}$ & $\mathrm{y}$ & 9220 & 3 & 1200 & 510 & 11 \\
\hline 8 & 40 & 10 & 500 & Simultaneous & $\mathrm{W}, \mathrm{s}, \mathrm{X}, \mathrm{Z}$ & $\mathrm{y}$ & 18440 & 3 & 2400 & 510 & 15 \\
\hline 9 & 30 & 20 & 1000 & Simultaneous & $\mathrm{W}, \mathrm{s}, \mathrm{X}, \mathrm{Z}$ & $\mathrm{y}$ & 13830 & 3 & 1800 & 1020 & 22 \\
\hline 10 & 20 & 10 & 500 & Decomposition & $\mathrm{W}, \mathrm{s}, \mathrm{X}, \mathrm{Z}$ & $\mathrm{y}$ & 9220 & 3 & 1200 & 510 & 28 \\
\hline 11 & 40 & 10 & 500 & Decomposition & $\mathrm{W}, \mathrm{s}, \mathrm{X}, \mathrm{Z}$ & $\mathrm{y}$ & 18440 & 3 & 2400 & 510 & 58 \\
\hline 12 & 30 & 20 & 1000 & Decomposition & $\mathrm{W}, \mathrm{s}, \mathrm{X}, \mathrm{Z}$ & $\mathrm{y}$ & 13830 & 3 & 1800 & 1020 & 76 \\
\hline
\end{tabular}

Table 4. Summary of SAA results for different sample sizes, using cost as objective function (see Section 6.2 for the explanation of the SAA estimators)

\begin{tabular}{|c|c|c|c|c|c|c|}
\hline Configuration & $\begin{array}{c}\bar{v}_{N, M} \\
(\$ \mathrm{~m})\end{array}$ & $\sigma_{\bar{v}_{N, M}}^{2}$ & $\widetilde{f}_{N^{\prime}}(\bar{y})$ & $\sigma_{N^{\prime}}^{2}(\bar{y})$ & gap $_{N_{N}, N^{\prime}}$ & $\sigma_{\text {gap }}^{2}$ \\
\hline 1 & 29.343 & 0.0350 & 29.5610 & 0.00606 & $0.74 \%$ & 0.0420 \\
\hline 2 & 29.460 & 0.0041 & 29.6600 & 0.01180 & $0.67 \%$ & 0.0160 \\
\hline 3 & 29.473 & 0.0087 & 29.7160 & 0.01040 & $0.82 \%$ & 0.0192 \\
\hline 4 & 29.414 & 0.0200 & 29.5485 & 0.00400 & $0.05 \%$ & 0.0240 \\
\hline 5 & 29.468 & 0.0086 & 29.6930 & 0.00440 & $0.76 \%$ & 0.0131 \\
\hline 6 & 29.463 & 0.0057 & 29.6810 & 0.00220 & $0.73 \%$ & 0.0080 \\
\hline 7 & 29.248 & 0.0118 & 29.4220 & 0.00640 & $0.59 \%$ & 0.0182 \\
\hline 8 & 29.414 & 0.0246 & 29.5395 & 0.00400 & $0.43 \%$ & 0.0287 \\
\hline 9 & 29.412 & 0.0230 & 29.5743 & 0.00230 & $0.55 \%$ & 0.0253 \\
\hline 10 & 29.237 & 0.0185 & 29.5163 & 0.02130 & $0.94 \%$ & 0.0398 \\
\hline 11 & 29.281 & 0.0110 & 29.4187 & 0.00440 & $0.46 \%$ & 0.0154 \\
\hline 12 & 29.290 & 0.0037 & 29.4183 & 0.00594 & $0.43 \%$ & 0.0097 \\
\hline
\end{tabular}




\subsection{Integration of SAA and $\varepsilon$-constraint approaches}

Once the multiobjective model has been converted into a single-objective optimization problem and a suitable solution method for the SAA problem chosen (as in Section 6.2), our integrated approach can proceed. Its implementation is discussed in this section.

\section{Proposed algorithm:}

1. define $\delta, l b$ and $u b$ for $\varepsilon$ and allowed optimality gap

2. $\varepsilon=l b$

3. define SAA parameters $N, M$ and $N^{\prime}$

4. repeat

5. solve SAA problem

6. $\quad$ while optimality gap > allowed gap

7. $\quad$ redefine SAA parameters $N, M$ and $N^{\prime}$

$8 . \quad$ solve SAA problem

9. $\quad$ end

10. $\quad$ save solution

11. $\varepsilon=\varepsilon+\delta$

12. until $\varepsilon>u b$

13. eliminate weak solutions and generate Pareto front

Here $\delta$ denotes the step value (increment) for $\varepsilon$ and $l b, u b$ are respectively the lower and upper limits for $\varepsilon$.

The first step of the algorithm defines the parameters used during execution. In particular, the integrated approach requires some experimentation to determine the limits of the $\varepsilon$-constraint algorithm. Given the stochastic nature of the problem, these limits cannot be defined directly by lexicographic solution of the deterministic model as in Table 2. For example, in our case we are using a triangular distribution to generate the samples of the scenarios. If we used the minimum value of this distribution as a lower value for $\varepsilon$ this would generate many infeasible models, i.e. models where the number of stockouts is unacceptably large. It is necessary to experiment in order to define an acceptable starting lower bound for the augmented $\varepsilon$-constraint algorithm. This lower bound will depend on the number of stockouts acceptable to the decision-maker. In our case, we started by solving an SAA model with a very low value of $\varepsilon$ (with a high number of stockouts) and gradually increased it until no stockouts occurred in the solution. We used this value as the lower limit $l b$. Once an acceptable lower level has been determined, the augmented $\varepsilon$-constraint algorithm can be run over the interval between this calculated lower limit and the maximum number of donors described in Table 2. In this particular case, the lower limit is found to be 148,000 donors and the upper limit, 190,600 donors. It would also be possible to build the Pareto front by intervals, switching between the objective functions at some point on the Pareto front. In this case, the solution defines the limits implicitly. It would also be possible to generate the 
Pareto front by starting from a central point and then varying the $\varepsilon$ value in both directions until the limits become evident. Finally, we note that the Pareto front generated by any of these methods is an estimator for the "true" Pareto front, and is presented alongside the optimality gap.

It is necessary to define the parameters to run each SAA problem. The parameters include the size of the sample $N$, the number of replications $M$, and the size of the independent sample $N^{\prime}$ that is used to evaluate the solution chosen from the $M$ replications. Section 6.2 presented experiments to find both a suitable solution method and the parameter configuration for one instance of the SAA problem. We use this configuration as the default for solving the SAA problems for all $\varepsilon$ values. However, it should be noted that our algorithm evaluates the convergence for each individual $\varepsilon$ value; this means that the initial configuration could be modified if the optimality gap were higher than allowed ( $1 \%$ for all the $\varepsilon$ values). In that case the sample size $N$ could be increased and the SAA model solved again until the optimality gap conditions are met, or alternatively the allowed optimality gap could be redefined for that particular instance of $\varepsilon$.

Computation time can be a disadvantage of our integrated approach, since at least one SAA model must be run for every value of $\varepsilon$, depending on the convergence rate of the solutions. For example, to evaluate $100 \varepsilon$ values, each of which only requires one SAA model, with parameters $N, M$ and $N^{\prime}$ equal to 30 , 20 and 1,000 respectively (configuration 9 in Tables 3 and 4), we would have to solve 102,000 singleobjective models. For each value of $\varepsilon$, we first need to solve $M=20$ two-stage stochastic programming models, each of which simultaneously includes $N=30$ scenarios. After this, $N^{\prime}=1000$ deterministic models are run in order to evaluate the best solution from these 20 models. Hence a total of 1020 models are run for each $\varepsilon$. We note that the first 20 models are considerably more complex to solve, given the large number of variables associated with the 30 scenarios. The actual number of models to be solved will depend on how fast the stochastic problem converges, and the level of accuracy desirable for the Pareto front. Our approach can generate robust decision support, but if the solution time of the SAA problem is too long, the total execution time would be prohibitive. Table 5 presents the parameters for executing the integrated approach, for three settings which differ according to the number of $\varepsilon$ values to be evaluated and the results generated. The first setting presents the evaluation of $40 \varepsilon$ values; in this case, the run time is shorter but only 25 optimal solutions are obtained. In contrast, Setting 3 presents the evaluation of $100 \varepsilon$ values, increasing the execution time but generating a more accurate representation of the Pareto front. 
Table 5. Parameter configuration and solutions generated for configuration 9

\begin{tabular}{|l|c|c|c|}
\hline & Setting 1 & Setting 2 & Setting 3 \\
\hline SAA solution strategy (see Tables 3 and 4) & \multicolumn{3}{|c|}{ configuration 9 } \\
\hline Number of $\varepsilon$ values & 40 & 60 & 100 \\
\hline Models solved & 40,800 & 61,200 & 102,000 \\
\hline Optimal solutions found & 25 & 32 & 62 \\
\hline Execution time (min) & 19.5 & 32.5 & 52.1 \\
\hline
\end{tabular}

In order to solve these models, the problems were coded using the Gurobi Java Interface and solved using Gurobi solver, version 5.3.6. The SAA and augmented $\varepsilon$-constraint procedures were also implemented using Java as the language for controlling the algorithm. The computational experiments were performed in a PC running Windows 7, with 6 GB of RAM memory and an i5 processor.

\section{Results}

The Pareto front presented in Figure 2 contains the set of efficient solutions obtained using Setting 3 in Table 5; i.e. it has been generated by evaluating $100 \varepsilon$ values and using 30, 20 and 100 as $N, M$ and $N^{\prime}$ respectively. The initial solution method for each SAA problem is described in configuration 9 from Tables 3 and 4.

Using these results for the Pareto front, the decision-maker can analyse the impact of a given maximum cost on the minimum number of donors needed, or vice versa. If some region of the Pareto front is of particular interest, the decision-maker can re-set the limits of the algorithm and run it again in order to obtain solutions with greater precision. However, it should be noted that in the real world, final decisions must consider other factors such as the capacity to motivate donors for the different collection methods and the preferences of physicians for each collection method. 


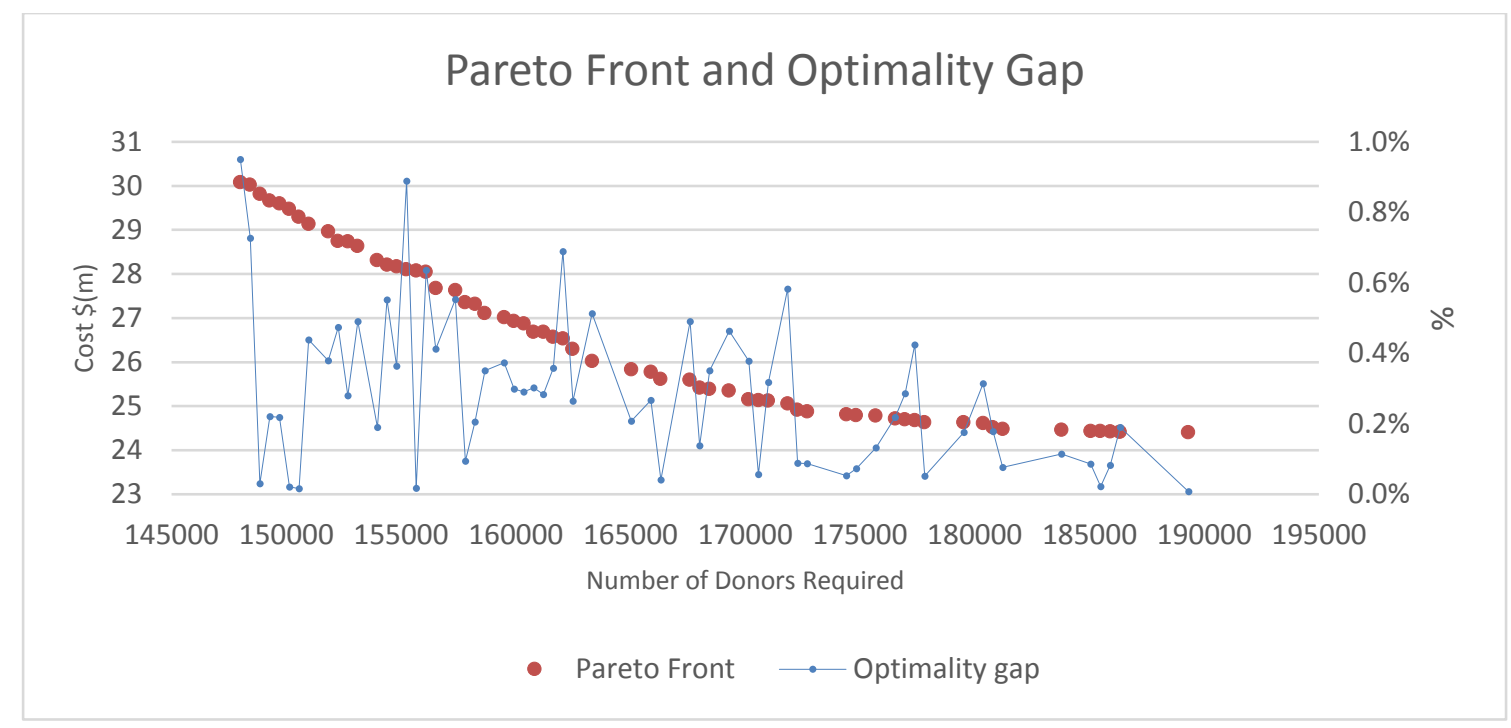

Figure 2. Pareto front for the multi-objective stochastic model presented

In Figure 2, the red dots represent the Pareto front; each dot shows the value for the two objective functions, the average number of donors (given an epsilon value) and an estimator for the true value of the objective function cost obtained using the sample $N^{\prime}$. In addition, the blue line shows the optimality gap for each solution obtained. All the solutions have an optimality gap lower than $1 \%$ which is a good indicator of convergency. The average optimality gap is $0.3 \%$.

The model also provides the optimal allocation of donors to different collection methods. Since these differ in terms of efficiency and cost, the specific allocation of donors to each method will also be affected by constraints on cost or the maximum number of donors required. Figure 3 presents the percentage of donors allocated to each collection method for each solution of the Pareto front.

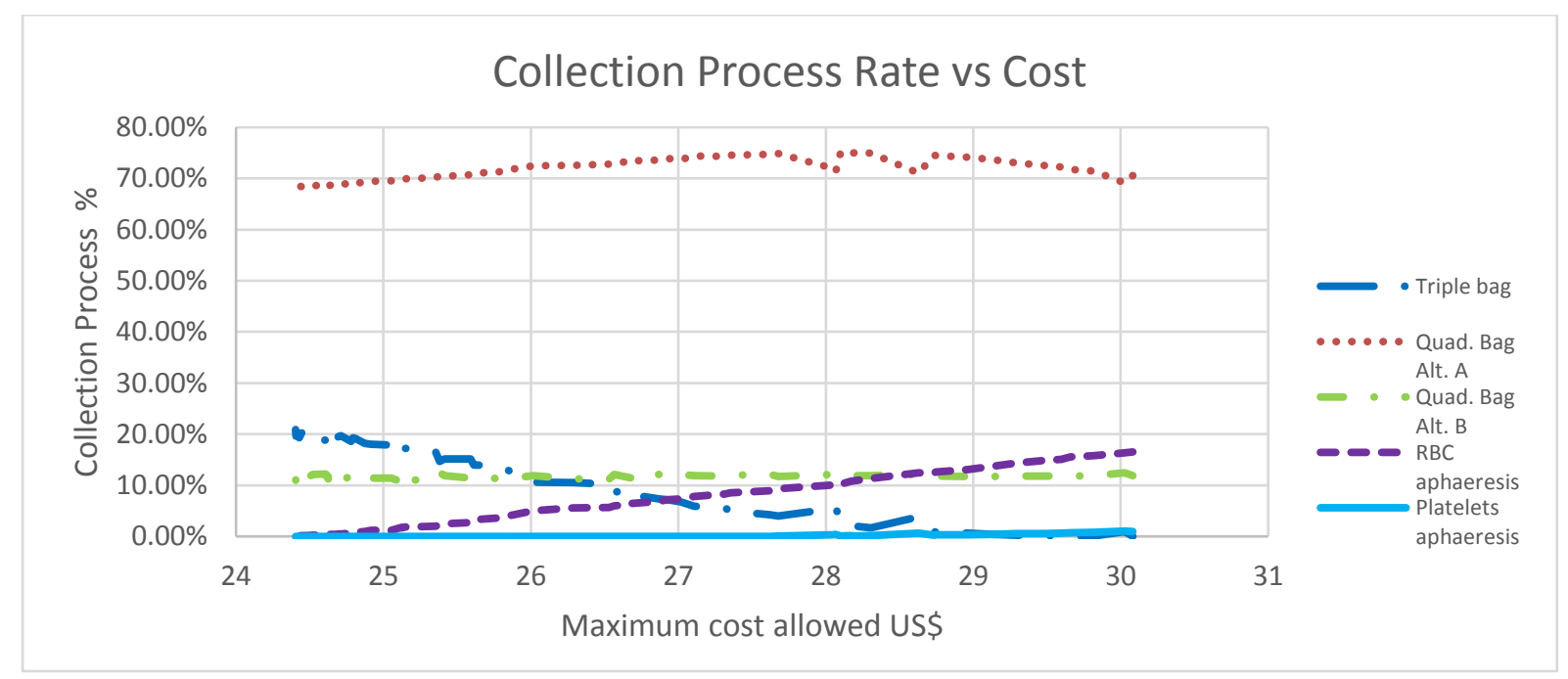

Figure 3. Fraction of donors allocated to each collection method 
As expected, the proportion of donors assigned to apheresis collection methods increases when the maximum permitted cost is higher, but this also means that fewer donors are required, based on the Pareto front. By combining the information from Figures 2 and 3, the decision-maker can examine trade-offs and determine the optimal donor allocation for each collection method when both objectives are optimized.

Finally, once the decision-maker has selected a suitable point on the Pareto front with which s/he is satisfied, the solutions of the associated stochastic optimization problems at this point provide additional and more detailed information, such as donor allocations for specific blood groups and the use of substitute products. For example, Table 6 and Appendix D show the solution obtained when the maximum number of donors is set to 155,000 . Table 6 presents the allocation of donors to the different collection processes, by blood type. The solution conserves the proportionality of blood types in the population of Bogota. The results presented in Table 6 correspond to the demand structure of this particular case. The solutions also provide information on the use of substitute products: Tables D.1 to D.4 in Appendix D present details about the allocation of each product to meet the demand for other products.

Table 6. Donor allocation to each process for a maximum number of donors of 155,000

\begin{tabular}{|l|r|r|r|r|r|r|r|r|r|}
\hline Process & \multicolumn{1}{|c|}{ A- } & A+ & AB- & AB+ & B- & B+ & O- & O+ & Total \\
\hline Triple bag & 28 & 783 & 8 & 324 & 4 & 1382 & 16 & 1397 & $\mathbf{3 9 4 2}$ \\
\hline Quadruple bag - Alternative A & 3167 & 31822 & 378 & 1326 & 749 & 7423 & 5674 & 65884 & $\mathbf{1 1 6 4 2 3}$ \\
\hline Quadruple bag - Alternative B & 428 & 6472 & 78 & 617 & 162 & 2046 & 235 & 8402 & $\mathbf{1 8 4 4 0}$ \\
\hline 2-RBC (RBC by apheresis) & 522 & 1391 & 8 & 12 & 154 & 427 & 1983 & 11589 & $\mathbf{1 6 0 8 6}$ \\
\hline Platelets by apheresis & 44 & 8 & 13 & 4 & 21 & 11 & 32 & 13 & $\mathbf{1 4 6}$ \\
\hline Total & $\mathbf{4 1 8 9}$ & $\mathbf{4 0 4 7 6}$ & $\mathbf{4 8 5}$ & $\mathbf{2 2 8 3}$ & $\mathbf{1 0 9 0}$ & $\mathbf{1 1 2 8 9}$ & $\mathbf{7 9 4 0}$ & $\mathbf{8 7 2 8 5}$ & $\mathbf{1 5 5 0 3 7}$ \\
\hline
\end{tabular}

Note that the total number of donors in Table 6 is slightly greater than 155,000 , the epsilon value of the SAA execution. This is because the decision variables were initially relaxed and later rounded up in order to obtain an integer solution. In practice, the decision-maker would need to consider whether such a small difference in donor numbers was significant, or whether the results would need to be re-scaled.

The model and the results can be interpreted and used in several ways. Firstly, the model gives the optimal configuration of equipment to meet demand. In addition, it provides an optimal tactical collection and production strategy. The planning horizon used in the model is one year, but the results can be broken down by month or week to establish collection goals and determine a production plan. The model also includes the use of substitute products. Finally, the model could be used for different periods and situations. For example, it can be easily modified to evaluate the best collection strategy for a short time period given a pre-defined capacity. 


\section{Conclusions and further research}

Decision-making in uncertain environments is a complex task. The methodology used to generate robust solutions for problems with stochastic parameters will depend both on the problem features and on the resources available. Since the model presented in this paper is linear and its deterministic version is easy to solve using an optimization package, we have developed an integrated approach using the SAA methodology combined with the augmented $\varepsilon$-constraint. This approach generates robust solutions that consider the stochastic nature of demand and also guarantee that features such as proportionality and compatibility are met.

The blood supply chain has been widely studied, but previous research has focused mainly on the development of inventory policies. The model presented in this paper addresses decision stages which have received less attention, i.e. collection and production. Moreover, the model considers multiple products, instead of focusing on individual products, since fractionation processes always generate more than one product.

In practice the optimal collection strategy will depend on several factors such as demand structure, physician preferences, donor response to different collection methods and the maximum cost allowed. However, robust methodologies such as ours support real-world decision-making in the blood supply chain, presenting a wide view of the impact of choosing any given strategy. In this particular study, the relationship between the number of donors required and cost is presented. In reality, making optimal decisions considering all the blood supply chain aspects such as compatibilities and proportionalities is almost impossible without using advanced decision-making methodologies.

Our model can easily be used by blood centres to plan their collection strategy, since the necessary data is usually readily available in blood centre information systems. The model assumes the use of substitute products, but different substitution rules can be easily applied by changing the compatibility parameters. We are keen for our model to be used in practice. However for this to happen it needs to be implemented in software that blood centre managers can use and, especially in the case of developing countries, is relatively inexpensive. The model in this paper was solved using the Gurobi solver, but other efficient solvers are available and can be integrated into spreadsheets to facilitate their implementation and use in practice. A similar version of our model was implemented in Excel and solved using Open Solver. It was tested using data from one of the largest blood centres in Colombia in order to obtain their annual plans.

There are many ways in which this model can be extended. We have included only the most common blood products and production processes, but there are numerous other products such as irradiated or washed products that could be considered. There are also other collection and fractionation alternatives apart from those shown in Table 1. Examples of methods used in other countries include whole blood 
without fractionation, double bag, and plasma apheresis. Another possible extension is the inclusion of decisions for purchasing blood products from external suppliers, in settings where this is permitted. It would be possible to study the best way to meet demand, including both internal and external sources of supply. Finally, the inclusion of different types of demand and preferences on compatibilities could also be easily modelled.

\section{Acknowledgements}

We are grateful for the comments of the editor and anonymous reviewers, which have greatly improved the quality of this paper.

The first author's research is funded by a $\mathrm{PhD}$ scholarship from the Departamento Administrativo de Ciencia y Tecnologia, Colciencias, Bogota, Colombia.

\section{References}

Alfonso, E., Xie, X.L., Augusto, V. and Garraud, O. (2012) Modeling and simulation of blood collection systems. Health Care Management Science, 15 (1), 63-78.

Alfonso, E., Xie, X.L., Augusto, V. and Garraud, O. (2013) Modelling and simulation of blood collection systems: Improvement of human resources allocation for better cost-effectiveness and reduction of candidate donor abandonment. Vox Sanguinis, 104 (3), 225-233.

Arciniegas, A. and Mosquera, M. (2012) [Model for Production Planning of Blood Components in a Blood Bank in Cali], Industrial Engineer, Icesi University. (In Spanish).

Baesler, F., Martinez, C., Yaksic, E. and Herrera, C. (2011) Logistic and production process in a regional blood center: modeling and analysis. Revista Medica de Chile, 139 (9), 1150-1156.

Baesler, F., Nemeth, M., Martínez, C. and Bastías, A. (2013) Analysis of inventory strategies for blood components in a regional blood center using process simulation. Transfusion. Available from: http://dx.doi.org/10.1111/trf.12287 [Accessed 4 November 2013].

Bérubé, J.-F., Gendreau, M. and Potvin, J.-Y. (2009) An exact -constraint method for bi-objective combinatorial optimization problems: Application to the Traveling Salesman Problem with Profits. European Journal of Operational Research, 194 (1), 39-50.

Boppana, R.V. and Chalasani, S. (2007) Analytical models to determine desirable blood acquisition rates. Paper presented at 2007 IEEE International Conference on System of Systems Engineering, San Antonio, TX, 16-18 April 2007. Available from: http://ieeexplore.ieee.org/stamp/stamp.jsp?tp=\&arnumber=4304327 [Accessed 3 December 2013].

Cardona-Valdés, Y., Álvarez, A. and Ozdemir, D. (2011) A bi-objective supply chain design problem with uncertainty. Transportation Research Part C: Emerging Technologies, 19 (5), 821-832.

Chazan, D. and Gal, S. (1977) A Markovian model for a perishable product inventory. Management Science, 23 (5), 512-521.

Cohen, M.A. (1976) Analysis of single critical number ordering policies for perishable inventories. Operations Research, 24 (4), 726-741.

Cumming, P.D., Kendall, K.E., Pegels, C.C., Seagle, J.P. and Shubsda, J.F. (1976) A collections planning model for regional blood suppliers: description and validation. Management Science, 22 (9), 962-971. 
Du, Y., Xie, L., Liu, J., Wang, Y., Xu, Y. and Wang, S. (2014) Multi-objective optimization of reverse osmosis networks by lexicographic optimization and augmented epsilon constraint method. Desalination, 333 (1), 66-81.

Duan, Q. and Liao, T.W. (2013) A new age-based replenishment policy for supply chain inventory optimization of highly perishable products. International Journal of Production Economics, 145 (2), 658-671.

Duan, Q. and Liao, T.W. (2014) Optimization of blood supply chain with shortened shelf lives and ABO compatibility. International Journal of Production Economics, (0).

Ehrgott, M. and Ruzika, S. (2008) Improved $\varepsilon$-Constraint Method for Multiobjective Programming. Journal of Optimization Theory and Applications, 138 (3), 375-396.

Fonseca, M.C., García-Sánchez, Á., Ortega-Mier, M. and Saldanha-da-Gama, F. (2010) A stochastic biobjective location model for strategic reverse logistics. TOP, 18 (1), 158-184.

Glynn, S.A., Busch, M.P., Schreiber, G.B., Murphy, E.L., Wright, D.J., Tu, Y. and Kleinman, S.H. (2003) Effect of a national disaster on blood supply and safety: The September 11 experience. JAMA, 289 (17), 2246-2253.

Gutjahr, W. and Pichler, A. (2013) Stochastic multi-objective optimization: a survey on non-scalarizing methods. Annals of Operations Research, 1-25. Available from: http://dx.doi.org/10.1007/s10479-013-1369-5.

Haimes, Y., Lasdon, L. and Wismer, D. (1971) On a Bicriterion Formulation of the Problems of Integrated System Identification and System Optimization. IEEE Transactions on Systems, Man, and Cybernetics, 1 (3), 296-297.

Hemmelmayr, V., Doerner, K.F., Hartl, R.F. and Savelsbergh, M.W.P. (2010) Vendor managed inventory for environments with stochastic product usage. European Journal of Operational Research, $202(3), 686-695$.

INS (2013) Informe Nacional de Indicadores 2012 [National Report of Indicators 2012]. Instituto Nacional de Salud. Available from: http://www.ins.gov.co/lineas-de-accion/Red-NacionalLaboratorios/reas\%20Estratgicas/Informe\%20anual\%20Red\%20Sangre\%202012.pdf. [Accessed November 20 2013].

Jagannathan, R. and Sen, T. (1991) Storing crossmatched blood: a perishable inventory model with prior allocation. Management Science, 37 (3), 251-266.

Katsaliaki, K. and Brailsford, S.C. (2007) Using simulation to improve the blood supply chain. Journal of the Operational Research Society, 58 (2), 219-227.

Kiya, F. and Davoudpour, H. (2012) Stochastic programming approach to re-designing a warehouse network under uncertainty. Transportation Research Part E: Logistics and Transportation Review, 48 (5), 919-936.

Kleywegt, A., Shapiro, A. and Homem-de-Mello, T. (2002) The Sample Average Approximation Method for Stochastic Discrete Optimization. SIAM Journal on Optimization, 12 (2), 479-502.

Li, P.-Y. (2014) Sample average approximation method for a class of stochastic generalized Nash equilibrium problems. Journal of Computational and Applied Mathematics, 261 (0), 387-393.

Lowalekar, H. and Ravichandran, N. (2010) Model for blood collections management. Transfusion, 50 (12pt2), 2778-2784.

Madden, E., Murphy, L. and Custer, B. (2007) Modeling red cell procurement with both double-redcell and whole-blood collection and the impact of European travel deferral on units available for transfusion. Transfusion, 47 (11), 2025-2037.

Marler, R.T. and Arora, J.S. (2004) Survey of multi-objective optimization methods for engineering. Structural and Multidisciplinary Optimization, 26 (6), 369-395.

Mavrotas, G. (2009) Effective implementation of the $\varepsilon$-constraint method in Multi-Objective Mathematical Programming problems. Applied Mathematics and Computation, 213 (2), 455465 . 
Melnyk, S.A., Pagell, M., Jorae, G. and Sharpe, A.S. (1995) Applying survival analysis to operations management: Analyzing the differences in donor classes in the blood donation process. Journal of Operations Management, 13 (4), 339-356.

Nahmias, S. and Pierskalla, W.P. (1976) A two-product perishable/nonperishable inventory problem. SIAM Journal on Applied Mathematics, 30 (3), 483-500.

Ogbe, E. and Li, X. (2017) A new cross decomposition method for stochastic mixed-integer linear programming. European Journal of Operational Research, 256 (2), 487-499.

Osorio, A.F., Brailsford, S. and Smith, H. (2014) [A bi-objective optimization model for technology selection and donor's assignment in the blood supply chain]. Sistemas y Telemática, 12 (30), 9-24. (In Spanish).

Osorio, A.F., Brailsford, S.C. and Smith, H.K. (2015) A structured review of quantitative models in the blood supply chain: a taxonomic framework for decision-making. International Journal of Production Research, 53 (24) 7191-7212.

Pierskalla, W.P. and Roach, C.D. (1972) Optimal issuing policies for perishable inventory. Management Science, 18 (11), 603-614.

Rytilä, J.S. and Spens, K.M. (2006) Using simulation to increase efficiency in blood supply chains. Management Research News, 29 (12), 801-819.

Santoso, T., Ahmed, S., Goetschalckx, M. and Shapiro, A. (2005) A stochastic programming approach for supply chain network design under uncertainty. European Journal of Operational Research, 167 (1), 96-115.

Schütz, P., Tomasgard, A. and Ahmed, S. (2009) Supply chain design under uncertainty using sample average approximation and dual decomposition. European Journal of Operational Research, 199 (2), 409-419.

SDS (2013) Boletín Estadístico, Red Distital de Sangre 2012 [Statistical Bulletin, Distrital Blood Network 2012]. Secretaria Distrital de Salud. Available from: http://www.saludcapital.gov.co/DDS/Boletin\%20Estadistico/BOLETIN\%20ESTAD\%C3\%8DSTI CO\%20RED\%20DE\%20SANGRE\%202012.pdf. [Accessed November 20 2013].

Seifried, E., Klueter, H., Weidmann, C., Staudenmaier, T., Schrezenmeier, H., Henschler, R., Greinacher, A. and Mueller, M.M. (2011) How much blood is needed? Vox Sanguinis, 100 (1), 10-21.

Simonetti, A., Forshee, R., Anderson, S. and Walderhaug, M. (2013) A stock-and-flow simulation model of the US blood supply. Transfusion. Available from: http://dx.doi.org/10.1111/trf.12392 [Accessed 28 November 2013].

Sonmezoglu, M., Kocak, N., Oncul, O., Ozbayburtlu, S., Hepgul, Z., Kosan, E., Aksu, Y. and Bayik, M. (2005) Effects of a major earthquake on blood donor types and infectious diseases marker rates. Transfusion Medicine, 15 (2), 93-97.

Toro-Diaz, H. and Osorio-Muriel, A. (2012) Stochastic Optimization of a Cash Supply Chain IN: Mejía, G. and Velasco, N. (eds.) Production Systems and Supply Chain Management in Emerging Countries: Best Practices. Springer Berlin Heidelberg, 183-199.

Tricoire, F., Graf, A. and Gutjahr, W.J. (2012) The bi-objective stochastic covering tour problem. Computers \& Operations Research, 39 (7), 1582-1592.

Verweij, B., Ahmed, S., Kleywegt, A., Nemhauser, G. and Shapiro, A. (2003) The Sample Average Approximation Method Applied to Stochastic Routing Problems: A Computational Study. Computational Optimization and Applications, 24 (2-3), 289-333.

WHO (2014) Blood safety and availability. Available from: http://www.who.int/mediacentre/factsheets/fs279/en/ [Accessed April 1 2014].

WHO (2016) Voluntary unpaid blood donations must increase rapidly to meet 2020 goal. Available from: http://www.who.int/mediacentre/news/releases/2016/world-blood-donor-day/en/ . [Accessed September 2016].

Woodget, M. (2014) NHS Blood and Transplant, Annual Functional Report - Estates \& Facilities. Available

from: 
http://www.nhsbt.nhs.uk/download/board papers/jan14/annual functional report estate $\mathrm{s}$ and facilities.pdf [Accessed September 28 2015].

Zhou, A., Qu, B.-Y., Li, H., Zhao, S.-Z., Suganthan, P.N. and Zhang, Q. (2011) Multiobjective evolutionary algorithms: A survey of the state of the art. Swarm and Evolutionary Computation, 1 (1), 3249. 
Table A.1 Red blood cell compatibility

\begin{tabular}{|c|c|c|c|c|c|c|c|c|c|}
\hline & & \multicolumn{8}{|c|}{ Patient } \\
\hline & ABO Rh & A- & A+ & AB- & $\mathbf{A B +}$ & B- & B+ & O- & O+ \\
\hline \multirow{8}{*}{ 苛 } & A- & $\mathrm{x}$ & $\mathrm{x}$ & $\mathrm{x}$ & $\mathrm{x}$ & & & & \\
\hline & $\mathrm{A}+$ & & $\mathrm{x}$ & & $\mathrm{X}$ & & & & \\
\hline & AB- & & & $\mathrm{x}$ & $\mathrm{x}$ & & & & \\
\hline & $\mathrm{AB}+$ & & & & $\mathrm{X}$ & & & & \\
\hline & B- & & & $\mathrm{X}$ & $\mathrm{X}$ & $\mathrm{X}$ & $\mathrm{X}$ & & \\
\hline & $\mathrm{B}+$ & & & & $\mathrm{X}$ & & $\mathrm{X}$ & & \\
\hline & O- & $\mathrm{x}$ & $\mathrm{x}$ & $\mathrm{x}$ & $\mathrm{X}$ & $\mathrm{x}$ & $\mathrm{X}$ & $\mathrm{x}$ & $\mathrm{x}$ \\
\hline & $\mathrm{O}+$ & & $\mathrm{x}$ & & $\mathrm{X}$ & & $\mathrm{X}$ & & $\mathrm{x}$ \\
\hline
\end{tabular}

Table A.2 Plasma and cryoprecipitate compatibility

\begin{tabular}{|l|l|l|l|l|l|}
\hline & & \multicolumn{4}{|l|}{ Patient } \\
\hline & ABO & A & AB & B & O \\
\hline \multirow{2}{*}{} & $\mathrm{A}$ & $\mathrm{x}$ & & & $\mathrm{x}$ \\
\cline { 2 - 6 } & $\mathrm{AB}$ & $\mathrm{x}$ & $\mathrm{x}$ & $\mathrm{x}$ & $\mathrm{x}$ \\
\cline { 2 - 6 } & $\mathrm{B}$ & & & $\mathrm{x}$ & $\mathrm{x}$ \\
\cline { 2 - 6 } & $\mathrm{O}$ & & & & $\mathrm{x}$ \\
\hline
\end{tabular}

Table A.3 Platelet compatibility

\begin{tabular}{|c|l|c|c|}
\hline & \multicolumn{3}{|c|}{ Patient } \\
\hline \multirow{2}{*}{$\tilde{\Xi}$} & Platelet Type & Rh - & Rh + \\
\cline { 2 - 4 } & $\mathrm{Rh}-$ & $\mathrm{x}$ & $\mathrm{x}$ \\
\cline { 2 - 4 } & $\mathrm{Rh}+$ & & $\mathrm{x}$ \\
\hline
\end{tabular}




\section{Appendix B}

Formulation of the augmented $\varepsilon$-constraint algorithm (Mavrotas (2009))

A multi-objective optimization problem with $k$ objectives is usually expressed as follows:

Find:

$$
x=\left[x_{1}, x_{2}, \ldots, x_{n}\right],
$$

that minimizes

$$
F(x)=\left[F_{1}(x), F_{2}(x), \ldots, F_{k}(x)\right],
$$

subject to:

$$
g_{j}(x) \leq 0, \quad j=1,2, \ldots, m .
$$

In order to convert the multi-objective model defined in (A.1) - (A.3) into a single-objective model using the augmented $\varepsilon$-constraint algorithm, the formulation is modified as follows:

Find the same vector expressed in (A.1) that minimizes:

$$
F(x)=F_{1}(x)-\beta * \sum_{i=2}^{k} \frac{w_{i}}{r_{i}},
$$

subject to:

$$
\begin{aligned}
& F_{i}(x)+w_{i}=\varepsilon_{i}, \quad i=2, \ldots, k, \\
& g_{j}(x) \leq 0, \quad j=1,2, \ldots, m .
\end{aligned}
$$

In this formulation $w$ represents a surplus variable for each epsilon constraint. These variables are added to the objective function with very low coefficients $(\beta)$ in a manner that does not distort the objective function but improves the function associated with the epsilon constraint. Finally, the parameter $r_{i}$ is the range of the values for each objective function $F_{i}(x), i=2, \ldots, k$. These coefficients work as a normalization operator in order to sum the objective functions. 


\section{Appendix C}

\section{Benders' decomposition algorithm}

Benders' decomposition algorithm decomposes the model, creating a master problem associated with the first-stage variables and a set of sub-problems associated with the second-stage variables. When first-stage variables are fixed these sub-problems are usually easy to solve. The solution of the subproblem allows the generation of optimality cuts to be included in the master problem. The process iterates until the solution of both master and sub-problems converge. In the version presented in Santoso et al. (2005) and used in this paper, the algorithm is adapted to consider $N$ scenarios solving one subproblem for each scenario. The detail of the algorithm is explained as follows:

Initialization step. Let the lower bound, $l b=-\infty$ and the upper bound, $u b=+\infty$, respectively and set the iteration counter $b=0$. Let $\hat{y}$ denote the incumbent solution.

Step 1: Solve the master problem

$$
l b=f(y)=\sum_{t \in T} y_{t} F_{t}+\theta
$$

subject to:

$$
\begin{aligned}
& \mathrm{y}_{t}, q \in \mathbb{Z}^{+} \\
& \theta \geq a_{c} y+b_{c}, c=1, \ldots, b . \\
& q=\varepsilon
\end{aligned}
$$

Step 2: For $n=1, \ldots, N$., solve the sub-problems (17) - (23) corresponding to the $y^{b}$ and $\xi^{n}=\left(d^{n}\right)$. The problem is re-stated as follows:

$$
Q^{\prime}(y, q, \xi)=\operatorname{minimize} \sum_{i \in I} \sum_{k \in K} P_{k} x_{i k}+\sum_{l \in J} H_{l} s_{l}-\beta w
$$

subject to:

$$
\begin{aligned}
& \sum_{j \in J} z_{j l} O_{j l}+s_{l} \geq D_{l}(\xi)\left(1+\alpha_{l}\right), \quad l \in J \\
& \sum_{l \in J} z_{j l} \leq \sum_{i \in I} \sum_{k \in K} x_{i k} A_{i j k}, \quad j \in J \\
& \sum_{k \in K} x_{i k} \leq N_{i} \sum_{a \in I} \sum_{k \in K} x_{a k}, \quad i \in I
\end{aligned}
$$




$$
\begin{aligned}
& \sum_{i \in I} \sum_{k \in K} x_{i k} R_{k t} \leq C_{t} y_{t}, \quad t \in \mathrm{T} \\
& x_{i g} \leq \sum_{k \in K} x_{i k} B, \quad i \in I, g \in G \\
& \sum_{i \in I} \sum_{k \in K} x_{i k}+w=q
\end{aligned}
$$

where $\mu_{l}, \tau_{j}, \phi_{i}, \rho_{m}, \psi_{i}$ are the dual values for constraints (3) - (7) while $\lambda$ is the dual value for constraint (17). The objective function value of the current solution $y^{i}$ can be computed using the subproblem objective values as follows:

$$
\hat{f}_{N}\left(y^{b}\right)=F y^{b}+\frac{1}{N} \sum_{n=1}^{N} Q^{\prime}\left(y^{b}, q, \xi^{n}\right)
$$

if $\hat{f}_{N}\left(y^{b}, q\right)<u b$ then $u b=\hat{f}_{N}\left(y^{b}, q\right)$ and $\hat{y}=y^{b}$.

Step 3: If $u b-l b<\delta$, where $\delta \geq 0$ is the pre-specified gap tolerance, stop and return $y^{b}$ as the optimal solution and $u b$ as the optimal objective value; otherwise proceed to Step 4.

Step 4: For each for $n=1, \ldots, N$. , let $\mu_{l b}^{n}, \tau_{j b}^{i}, \phi_{i b}^{n}, \rho_{t b}^{n}, \psi_{i b}^{n}$ and $\lambda_{b}^{n}$ be the optimal dual solutions. Compute the objective value for the corresponding to $y^{b}$ and $\xi^{n}$ solved in step 2. Compute the optimality cuts coefficients:

$$
a_{b+1}=\frac{1}{N} \sum_{n=1}^{N} \sum_{t=1}^{T} C_{t} \rho_{t b}^{n}
$$

And

$$
b_{b+1}=\frac{1}{N} \sum_{n=1}^{N}\left(\lambda_{b}^{n} \varepsilon+\sum_{l=i}^{L} D_{l}^{n}\left(1+\alpha_{l}\right) \mu_{l b}^{n}\right)
$$

Let $b=b+1$ and go to Step 1 . 


\section{Appendix D}

The following tables show the numbers of units of blood products allocated from donors to recipients by blood group according to compatibility.

Table D.1 Number of units of RBCs allocated by blood group

\begin{tabular}{|c|c|c|c|c|c|c|c|c|c|c|}
\hline & \multicolumn{10}{|c|}{ Recipient (RBCs units) } \\
\hline & ABO Rh & A- & $\mathbf{A +}$ & AB- & $\mathbf{A B +}$ & B- & B+ & O- & $\mathbf{O +}$ & Total \\
\hline \multirow{9}{*}{ 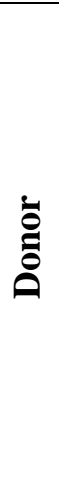 } & A- & 4253 & 374 & 35 & 5 & 0 & 0 & 0 & 0 & 4667 \\
\hline & $\mathbf{A +}$ & 0 & 41776 & 0 & 80 & 0 & 0 & 0 & 0 & 41856 \\
\hline & AB- & 0 & 0 & 449 & 29 & 0 & 0 & 0 & 0 & 478 \\
\hline & $\mathbf{A B +}$ & 0 & 0 & 0 & 2287 & 0 & 0 & 0 & 0 & 2287 \\
\hline & B- & 0 & 0 & 43 & 9 & 1025 & 146 & 0 & 0 & 1223 \\
\hline & B+ & 0 & 0 & 0 & 60 & 0 & 11642 & 0 & 0 & 11702 \\
\hline & O- & 376 & 113 & 8 & 8 & 179 & 87 & 8755 & 369 & 9895 \\
\hline & $\mathbf{O +}$ & 0 & 2287 & 0 & 47 & 0 & 628 & 0 & 95898 & 98860 \\
\hline & Total & 4629 & 44550 & 535 & 2525 & 1204 & 12503 & 8755 & 96267 & 170968 \\
\hline
\end{tabular}

Table D.2 Number of units of cryoprecipitates allocated by blood group

\begin{tabular}{|r|l|r|r|r|r|r|}
\hline \multirow{2}{*}{ ABO } & \multicolumn{5}{|c|}{ Recipient } \\
\cline { 2 - 7 } & A & AB & \multicolumn{1}{l|}{ B } & \multicolumn{1}{l|}{ O } & Total \\
\hline \multirow{4}{*}{} & A & 5272 & 0 & 0 & 1628 & $\mathbf{6 9 0 0}$ \\
\cline { 2 - 7 } & AB & 178 & 321 & 167 & 31 & $\mathbf{6 9 7}$ \\
\cline { 2 - 7 } & B & 0 & 0 & 1463 & 745 & $\mathbf{2 2 0 8}$ \\
\cline { 2 - 7 } & O & 0 & 0 & 0 & 8636 & $\mathbf{8 6 3 6}$ \\
\cline { 2 - 7 } & Total & $\mathbf{5 4 5 0}$ & $\mathbf{3 2 1}$ & $\mathbf{1 6 3 0}$ & $\mathbf{1 1 0 4 0}$ & $\mathbf{1 8 4 4 1}$ \\
\hline
\end{tabular}

Table D.3 Number of units of platelets allocated by platelet type

\begin{tabular}{|c|l|r|r|r|}
\hline \multicolumn{2}{|c|}{ Platelet Type } & \multicolumn{3}{c|}{ Recipient } \\
\cline { 3 - 5 } \multicolumn{2}{|c|}{} & Rh - & Rh + & \multicolumn{1}{c|}{ Total } \\
\hline \multirow{4}{*}{} & Apheresis Rh - & 953 & 107 & $\mathbf{1 0 6 0}$ \\
\cline { 2 - 5 } & Apheresis Rh + & 0 & 333 & $\mathbf{3 3 3}$ \\
\cline { 2 - 5 } & Fractionation Rh - & 9433 & 533 & $\mathbf{9 9 6 6}$ \\
\cline { 2 - 5 } & Fractionation Rh + & 0 & 106452 & $\mathbf{1 0 6 4 5 2}$ \\
\cline { 2 - 5 } & Total & $\mathbf{9 4 3 3}$ & $\mathbf{1 0 7 3 1 8}$ & $\mathbf{1 1 6 7 5 1}$ \\
\hline
\end{tabular}


Table D.4 Number of units of plasma allocated by blood group

\begin{tabular}{|r|l|l|r|l|r|r|}
\hline \multirow{2}{*}{ ABO } & \multicolumn{5}{|c|}{ Recipient } \\
\cline { 3 - 8 } & \multicolumn{1}{|l|}{ A } & \multicolumn{1}{l|}{ AB } & \multicolumn{1}{l|}{ B } & \multicolumn{1}{l|}{ O } & \multicolumn{1}{l|}{ Total } \\
\hline \multirow{4}{*}{} & A & 29621 & 0 & 0 & 6177 & $\mathbf{3 5 7 9 8}$ \\
\cline { 2 - 7 } & $\mathbf{A B}$ & 106 & 1721 & 173 & 36 & $\mathbf{2 0 3 6}$ \\
\cline { 2 - 7 } & $\mathbf{B}$ & 0 & 0 & 8403 & 1153 & $\mathbf{9 5 5 6}$ \\
\cline { 2 - 7 } & $\mathbf{O}$ & 0 & 0 & 0 & 72968 & $\mathbf{7 2 9 6 8}$ \\
\cline { 2 - 7 } & Total & $\mathbf{2 9 7 2 7}$ & $\mathbf{1 7 2 1}$ & $\mathbf{8 5 7 6}$ & $\mathbf{8 0 3 3 4}$ & $\mathbf{1 2 0 3 5 8}$ \\
\hline
\end{tabular}

